\title{
1 Where does value come from?
}

3 Keno Juechems* and Christopher Summerfield

4

5 Department of Experimental Psychology

6 University of Oxford

7 Walton Street, Oxford, UK

Abstract

The computational framework of reinforcement learning (RL) has allowed us to both understand biological brains and build successful artificial agents. However, in this article we highlight open challenges for $\mathrm{RL}$ as a model of animal behaviour in natural environments. We ask how the external reward function is designed for biological systems, and how we can account for the context sensitivity of valuation. We argue that rather than optimizing receipt of external reward signals, animals track current and desired internal states and seek to minimise the distance to goal across multiple value dimensions. Our framework can readily account for canonical phenomena observed in the fields of psychology, behavioural ecology, and economics, and recent findings from brain imaging studies of value-guided decision-making.

*Correspondence: keno.juchems@psy.ox.ac.uk

\section{Keywords}

Reinforcement learning; value; reward; goal-directed decision-making; medial prefrontal cortex 
What is it that animals seek to achieve by their behaviour? For evolutionary biologists, there is a simple answer to this question - that animal behaviour has evolved to maximise reproductive fitness, i.e. the propensity to produce offspring that will carry forth our genes. However, for those studying the behaviour of humans and animals in the fields of ethology, economics, psychology and neuroscience, this answer is only partly satisfying. Critically, it stops short of specifying how animals can learn which behaviours will increase or decrease their fitness. Instead, behavioural scientists propose that the environment furnishes reinforcement signals that indicate the likely costs or benefits of an action and assume that these signals can be directly sensed by the agent, allowing them to behave in ways that maximise utility over the short or long term. This is sometimes called the "reward hypothesis" [1].

Over the past half century, the fields psychology and artificial intelligence (AI) have jointly developed a normative theory, known as reinforcement learning (RL), that is founded on the reward hypothesis [3]. RL conceives of each encounter with the world as involving three distinct stages: (i) an agent receives sensory observations from the environment; (ii) the agent takes actions that influence its future states; and (iii) the agent receives a scalar reward signal that is emitted by the environment, and that is processed by the agent via a dedicated input channel (Fig. 1a). This conceptualisation lends itself naturally to modelling the canonical laboratory paradigm for operant behaviour, whereby an animal receives a sensory stimulus (e.g. a visual signal or tone), takes an action (e.g. presses a button or licks a spout) and receives a positive or negative reinforcer (e.g. liquid, food, or money). The framework successfully accounts for a wide array of habit-based and goal-directed behaviours in humans and other animals [4], and prominently explains the neural signals that accompany the expectation and delivery of reinforcers [5]. RL has furnished the canonical computational framework for understanding valueguided decision-making in humans and other primates, and has been widely used to explain how different brain regions participate in valuation and choice [3]. Moreover, RL models are currently offering some of the most exciting advances in Al research, producing artificial agents that can behave intelligently in complex, dynamic environments such as board and video games, thereby providing a proof of concept that intelligent behaviour can emerge de novo under the assumptions of the reward hypothesis $[6,7]$.

\section{The reward paradox}

The RL framework offers a useful set of computational tools for understanding animal learning and behaviour. However, as a theory of biological intelligence, it has a major shortcoming that often goes unacknowledged: it is unclear who designs the external reward function against which behaviour is optimised. In machine learning, the reward function for an RL model is hand-designed by the researcher. For example, when training an autonomous vehicle, she might specify that it needs to reach its destination as quickly as possible whilst obeying the traffic laws, by assigning benefits and costs for desired and undesired behaviours. However, in natural environments, no external entity exists that can directly quantify the consequences of each action, like the points that are awarded in a video game for completing levels or shooting monsters (Fig. 1b). Nor is it obvious that biological systems have a dedicated channel for receipt of external rewards that is distinct from the classical senses. Rather, rewards and punishments are sensory observations - the taste of an apple, the warmth of an embrace - and so stimulus value must be inferred by the agent, not conferred by the world. In other words, rewards must be intrinsic, not extrinsic (Box 1). We call this "the reward paradox". 
A
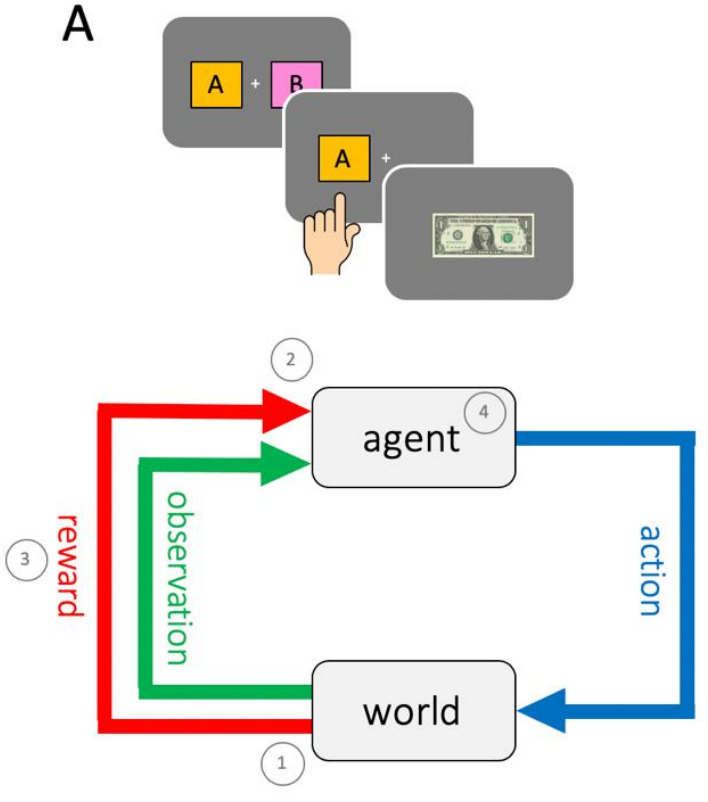

1. Rewards are "emitted" by the world

2. Rewards are sensed by the agent through dedicated input channels

3. Rewards are scalars (unidimensional signals)

4. Rewards are not fungible, but only exist to update the agent's value function
B

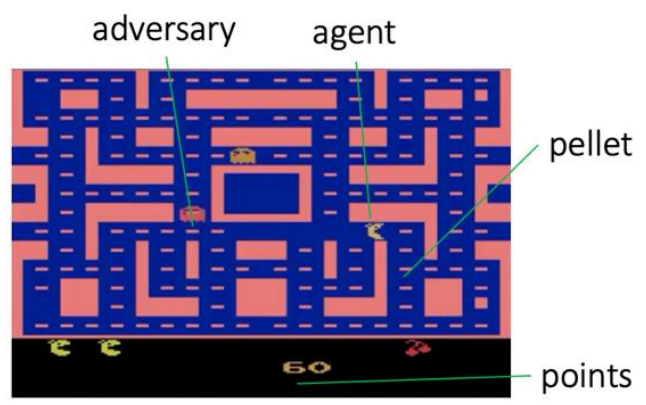

C

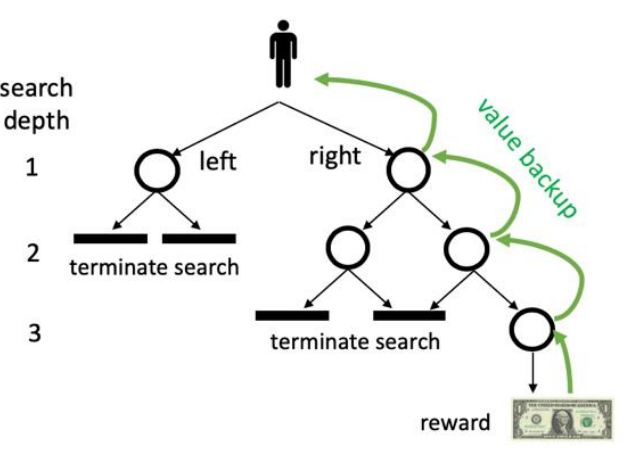

Figure 1. A. Schematic representation of the RL framework. Top: a typical "bandit" task in which participants choose between options ( $A$ and $B$ ) with uncertain reward probability, incurring financial outcomes. Below: in the $\mathrm{RL}$ framework, an agent receives observations (green), takes an action (blue) and receives feedback in the form of reward (red). The numbers highlight some assumptions of the RL framework that are poorly suited to explaining motivated behaviour in biological organisms (grey box below). B. The RL framework applied to video games (here, Ms. Pac-Man). The agent acts in order to maximise game score, which is a scalar quantity. Unlike humans playing the game, the agent receives the score differential directly (e.g. points for eating pellets) as reward signal via a dedicated input channel. C. Schematic of model-based RL, where the agent searches through a tree of possible future states and actions, sometimes stopping if no reward is expected from this branch of the search tree, and ultimately choosing the trajectory through the tree that leads to the highest return.

\section{Context-dependent valuation}

To circumvent the reward paradox, it is sometimes assumed that the reward function has been conveniently designed by evolution. In other words, reward computation may rely exclusively on fast, mandatory, phylogenetically prespecified mechanisms that automatically convert sensory observations into hedonic signals that act as a proxy for "external" rewards. Indeed, there is no doubt that dedicated mechanisms exist for those physiological drives that lie at the bottom of our "hierarchy of needs" [8], such as hunger or fatigue. These needs are regulated by projections from the hypothalamus to neural systems that respond to rewards, including dopaminergic neurons in the ventral tegmental area [9]. On this basis, proponents of RL models have dubbed dopamine neurons the "reward retina" as if they were responsible for directly sensing reinforcement from the environment [10].

However, one challenge for this perspective is that the reward value of a stimulus depends on the context in which it occurs. In other words, value depends on both the agent's internal state and the external environment. Intuitively, the value a potentially rewarding stimulus (e.g. a hamburger) will 
depend on the agent's ongoing bodily needs (am I hungry?). In the RL framework, there is no natural way to account for this context sensitivity. In fact, RL models assume that reward is a purely hedonic signal that is used solely to update the agent's value function. Reward is thus not fungible - that is, once acquired, it is not exchangeable for other assets, and cannot be used in a way that would alter future observations or facilitate future actions. For example, when RL agents are learning to play video games, the rewards they receive (i.e. game score differential) evaporate instantly without providing future opportunities for disbursement within the game. This is wholly unlike natural settings, where rewards are typically accumulated because they lead to persistent changes in state. For example, bodies act as repositories for energetic resources that are harvested by the agent during trophic behaviours, just as bank accounts are used to store for income acquired in exchange for work. The agent can thus choose to exchange one stored asset for another that is needed, the defining feature of economic behaviour in the marketplace. In the laboratory, rewards may be implicitly accumulated as experimental animals or human participants become more sated or wealthy as a result of the liquid rewards or financial incentives that they receive for performing the task, but these changes in state are typically considered to be nuisance variables by the experimenter. For example, when a monkey working for liquid reward is no longer thirsty enough to work, the researcher will typically terminate the experiment. To fully account for biological behaviour, our models of value learning need to account for the internal state of the organism.

Box 1. Intrinsic Motivation. Machine learning and Al researchers know that designing a reward function by hand
is costly and does not scale to real world settings. To avoid this problem, agents have been built that generate
their own intrinsic motivational signals [11-13]. For these agents, the cost function is populated by additional
terms that encourage the agent to pursue adaptive behaviours even in the absence of reward. Many of these
additional terms draw inspiration from psychological theory, which has long noted that humans and other animals
often pursue activities for their own sake (e.g. a kitten furiously chasing a piece of string, or a commuter
completing the crossword on her journey to work). For example, one key intrinsic motivation may be to explore
the world in a structured fashion, satisfying curiosity and avoiding boredom [14]. This allows agents to seek out
experiences that may accelerate learning without directly enhancing reward, a phenomenon known as active
learning. Other intrinsic motivation signals may encourage us to make actions with predictable consequences, or
that exert maximal control over the environment or the behaviour of conspecifics, or those that minimise
discrepant or dissonant inputs (see [11] for a review). Augmenting the reward function with these intrinsic
motivational signals often leads to faster learning and more stable patterns of behaviour in artificial agents [15].
However, in most reports, intrinsic motivation signals supplement (rather than replace) rewards from the external
environment, leaving the thorny question of where rewards come from only partially answered. Other
approaches that avoid any external feedback by using purely unsupervised or self-supervised methods - where
the agent is optimised to maximise information gain or reduce sensory surprise - hold undoubted promise but
have not yet been observed to yield complex behaviours in dynamic environments that resemble the natural
world [16].

Box 1. Intrinsic Motivation. Machine learning and Al researchers know that designing a reward function by hand is costly and does not scale to real world settings. To avoid this problem, agents have been built that generate their own intrinsic motivational signals [11-13]. For these agents, the cost function is populated by additional terms that encourage the agent to pursue adaptive behaviours even in the absence of reward. Many of these additional terms draw inspiration from psychological theory, which has long noted that humans and other animals often pursue activities for their own sake (e.g. a kitten furiously chasing a piece of string, or a commuter completing the crossword on her journey to work). For example, one key intrinsic motivation may be to explore the world in a structured fashion, satisfying curiosity and avoiding boredom [14]. This allows agents to seek out experiences that may accelerate learning without directly enhancing reward, a phenomenon known as active learning. Other intrinsic motivation signals may encourage us to make actions with predictable consequences, or that exert maximal control over the environment or the behaviour of conspecifics, or those that minimise discrepant or dissonant inputs (see [11] for a review). Augmenting the reward function with these intrinsic motivational signals often leads to faster learning and more stable patterns of behaviour in artificial agents [15]. However, in most reports, intrinsic motivation signals supplement (rather than replace) rewards from the external environment, leaving the thorny question of where rewards come from only partially answered. Other the agent is optimised to maximise information gain or reduce sensory surprise - hold undoubted promise but world [16]. 
A

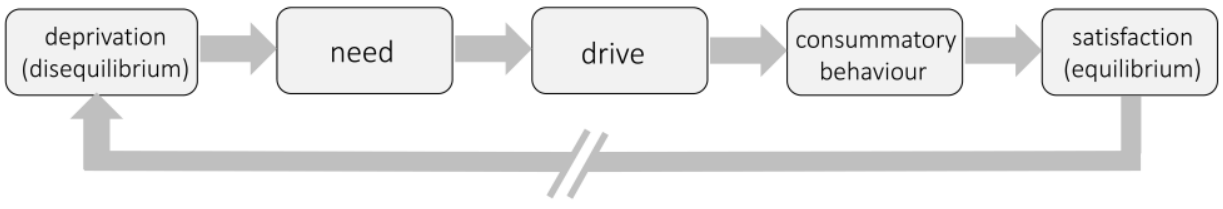

B

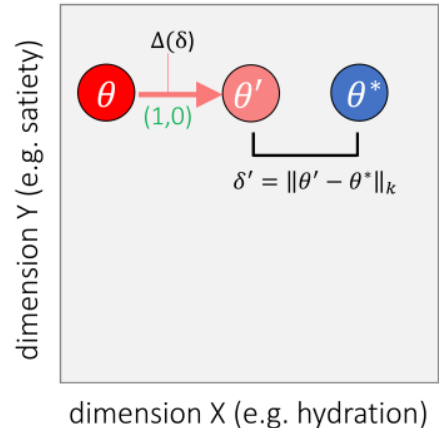

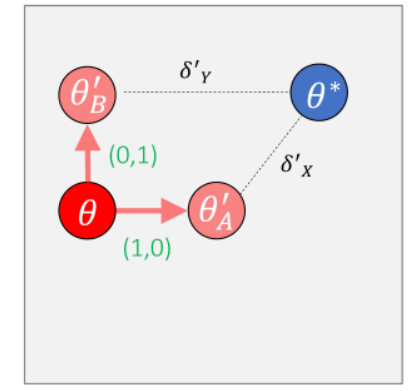

dimension X (e.g. hydration)

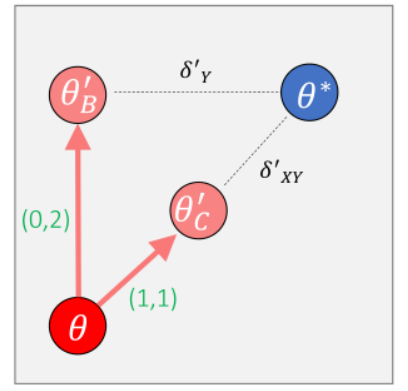

dimension X (e.g. hydration)

C

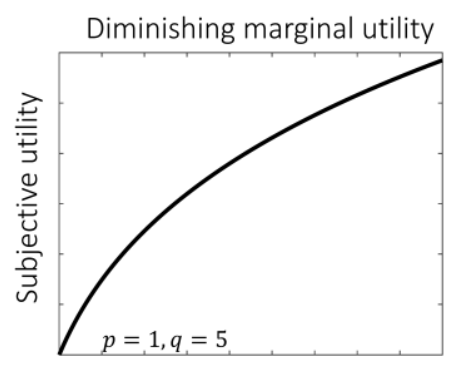

dimension $X$ (e.g. hydration)

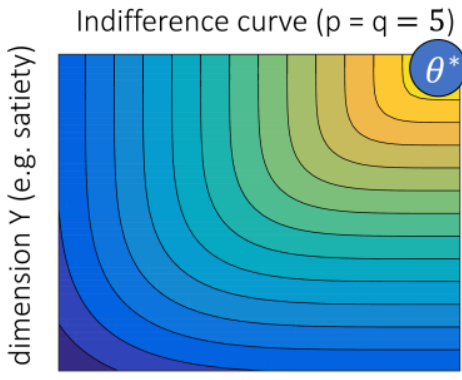

dimension X (e.g. hydration)

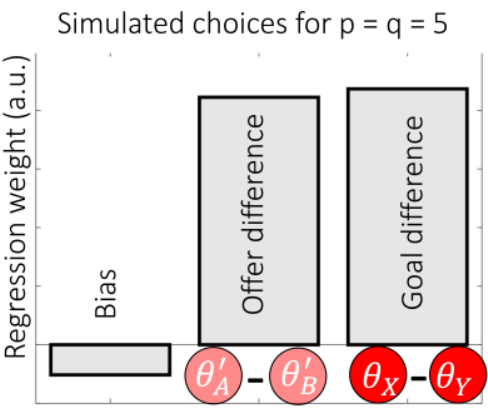

Figure 2. A. Schematic of Drive Reduction Theory. Behaviours are initiated to reduce drives, which in turn are provoked by needs that arise following homeostatic imbalance. B. Illustration of the Value Map, here in two example dimensions (labelled satiety and hydration). The agent's current state is denoted $\theta$, candidate next states $\theta^{\prime}$ and the desired state (setpoint) $\theta^{*}$. Offers in the $(x, y)$ dimension are shown in green. In the leftmost panel, the agent is sated but thirsty, as in a laboratory experiment where a monkey is working for liquid. An action reduces the distance $\delta$ between the current and desired state $\left\|\theta-\theta^{*}\right\|_{k}$ (where $k$ indicates the Minkowski distance; we use this here for simplicity, but note that a more general, two-parameter version depicted in panel $\mathrm{C}$ is needed to account for non-linearities in valuation, see Box 2 for details). Notice that the reduction in distance is proportional to what would (in the RL framework) be construed as an external reward signal. In the middle panel, an agent who is more thirsty than hungry is offered an equivalent quantity of food $(0,1)$ or water $(1,0)$. Water reduces distance to goal (Euclidean, $k=2$ ) more than food and is thus preferred. In the rightmost panel, the agent is both very hungry and very thirsty. An offer comprising a bundle of both food and water $(1,1)$ is preferred over a large quantity of food $(0,2)$. C. Predictions for the full model with two parameters, $p$ and $q$, governing non-linearities. The model can reproduce the ubiquitous finding of diminishing marginal utility (left panel). As $p$ and $q$ increase, distance estimates depend more strongly on just one dimension, indicating that maximum weight is given to the currently most needed asset (middle panel). Intermediate values $p$ and $q$ can reproduce one of the central findings of Juechems et al., 2019: that choices are both driven by offers and needs when assets need to be kept in equilibrium (see also Fig. 4). 


\section{The value of internal states}

A different theory has been invoked to understand how internal states motivate behaviour - one which assumes that animals seek to keep internal drives in equilibrium $[17,18]$. This theory builds on early models of homeostatic function [19] and found its first prominent expression in the 1940s with Hull's Drive Reduction Theory [20]. These homeostatic models argue that motivated behaviours seek to restore imbalance among different internal needs, such as warmth, satiety, and hydration (Fig. 2a). A central idea is that neural circuits encoded desired states or physiological "setpoints", and drives result from the disparity between current state and these setpoints. Thus, rewards are multidimensional quantities signalling the drive reduction that occurs when an imbalance among the organism's multiple internal needs is redressed. Pure drive reduction theories fell from favour with the realisation that animals will work for rewarding stimuli when their drives are satisfied, i.e. purely for their hedonic value $[21,22]$, which is important for understanding some psychological disorders, such as addiction. However, the incentive value of a stimulus nevertheless clearly depends on the internal state of the organism [23]. For example, the rate of approach towards food depends on hunger levels, and adapts immediately according to internal levels of satiety irrespective of prior training [24]. Similarly, food items that have been administered during states of food deprivation are preferred over control items during a later test when the animal is sated, suggesting they have acquired greater value [25]. Neither of these phenomena are readily accounted for by the RL framework.

Structure in ongoing or spontaneous brain states is a major driver of evoked neural activity [26], and neural signals across the brain may encode endogenous variation in internal motivational states. For example, genetic labelling can be used to identify neurons in the median preoptic nucleus of hypothalamus that become active when a mouse is thirsty, and optogenetic stimulation of these neurons provokes drinking behaviour even in hydrated mice [27]. Using large-scale recording methods to measure firing rates in multiple brain regions, every region tested (including neocortical regions such as the OFC) contained a substantial fraction of neurons that responded to thirst levels in the baseline period before a cue for water delivery was presented [28]. In other words, information about the animal's internal state of hydration is broadcast across the brain, in a way compatible with drive reduction theories. Thus, a more general framework is still needed in which value is computed from a combination of internal state and learned stimulus value [19]. This has been largely overlooked in cognitive theories of value learning which focus instead on the RL framework, where reward is wholly determined by the external reward function.

\section{Goals as cognitive setpoints}

In this Opinion we make a connection between homeostatic models of reward, which have emphasised balance among basic drives and interoceptive processes, and recent work on value-guided decisionmaking in humans and other primates [29-31]. We argue that motivated behaviour in cognitive, economic or social settings can similarly be understood as seeking to restore a balance among competing "setpoints" that correspond to higher-order goals. Unlike currently popular theories of value-guided choice, which are largely inspired by RL models [3] or expected utility theory from economics [32], our view emphasises the multidimensional nature of value signals, the dependence of motivated behaviour on the internal state of the agent, and the desire to explicitly realise goal states (constraint satisfaction) rather than maximise reward. In what follows, we show how this theory can explain some otherwise puzzling behavioural and neural findings in the literature on value-guided decision-making and economic choice. 
Motivated behaviour is intrinsically goal-directed in nature. Animals often appear to be driven by the attainment of discrete states, and the avoidance of others. This is particularly salient in humans, who will work tirelessly to complete tasks whose only apparent reward is the satisfaction of task completion itself, for example when seeking to win a race, solve a puzzle, or climb a mountain [33]. Indeed, this purposive, self-consistent property of human behaviour is so powerful that humans will seek to complete tasks even when the outcome is known to be a net loss, a phenomenon known as the sunk cost fallacy [34]. Although the RL framework embraces goal-directed behaviours, it does so under the assumption that animals explicitly learn the transition probabilities between states and use mental tree search to identify the most rewarding trajectories, which then guide "model-based" behaviours (see Fig. 1c). Thus, for RL, goals are not the objects of computation themselves but emerge as a by-product of policies which optimise expected future reward [35]. RL also offers no ready way to understand how abstract goals modulate rewards (a hamburger is worth less to a vegetarian than an omnivore). Similarly, affective states that may be potent modulators of value are notably absent from the RL framework, as when a hamburger is left uneaten by someone nervous before an exam. A complete theory of motivation needs to account for how value depends on goals and affective states as well as basic needs and drives.

We argue that during learning, animals form new setpoints pertaining to cognitive goals. For example, humans might represent current and desired states on axes pertaining to financial stability, moral worth, or physical health as well as hunger, thirst or temperature. A full computational model of this process is beyond the scope of the current article, but we offer a theory sketch in Box 2 . This sketch, which draws on extant models of homeostatic function [18], proposes that current states and goals are encoded in a multidimensional "value map". Motivated behaviour can then be seen as an attempt to minimise the maximum distance to setpoints in this value space. Agents commit to policies which focus on purposively driving the current state towards setpoints on a particular goal dimension, such as caching resources, building a shelter, obtaining a mate, or enhancing professional status. In doing so, their ultimate goal is to maintain equilibrium among all goal states, achieving what might be popularly characterised as a state of "wellbeing". We call this view "goal equilibrium theory".

In goal equilibrium theory, reward emerges naturally as a consequence of the computations required for learning, rather than being furnished by the external environment. This allows us to sidestep the "reward paradox" highlighted above. To illustrate, consider an animal whose internal state is currently described by parameters $\theta$ and who seeks to achieve a desired state $\theta^{*}$. Let us assume that the agent has a biologically plausible functional form, such as a neural network. In order to calculate the gradients that will allow network weights to be optimised for future behaviour, it is necessary to compute the loss term $\Delta\left(\left\|\theta^{*}-\theta\right\|_{k}\right)$, which indicates the change in distance between a current and desired state that is afforded by any action. Now consider a typical laboratory study in which a single reward dimension is relevant, for example because the experimenter reduces prior liquid intake and offers only water in exchange for behaviour. For a thirsty animal, this loss term is now mathematically identical to what one would construe as "reward" in the RL framework - i.e. the volume of liquid administered on a trial [18]. This equivalence will break down as $\theta$ tends towards $\theta^{*}$, but by this point the researcher will typically have ended the experiment because the animal is fully hydrated. Thus, goal equilibrium in this experiment can be thought of as subsuming the ubiquitous case were value is artificially constrained to be unidimensional. As an aside we note that from the agent's perspective, multiple value dimensions may continue to be relevant even during performance of a "unidimensional" value task. For example, a human participant might be tempted to neglect the task in favour of checking their social media account, in order to enhance social capital. However, this individual would most likely be excluded from the analysis. 


\section{Box 2: Goal equilibrium theory}

Goal equilibrium theory relies on three key intuitions. First, sensory observations, $\boldsymbol{X}_{\boldsymbol{t}}$ are mapped onto a persistent internal state $\boldsymbol{\theta}_{\boldsymbol{t}}$ :

$$
\theta_{t}=X_{t} \times U+\theta_{t-1} \times A_{\theta}
$$

Where $\boldsymbol{U}$ is a matrix mapping the chosen option $\boldsymbol{X}_{\boldsymbol{t}}$ onto $\boldsymbol{\theta}_{\boldsymbol{t}}$. The internal state, $\boldsymbol{\theta}_{\boldsymbol{t}}$, exhibits dynamics whereby it will change (decay) in the absence of any input, e.g. credit card debt will increase if it is not repaid, just as thirst will increase if not slaked. These dynamics are captured by the matrix $\boldsymbol{A}_{\boldsymbol{\theta}}$, which governs the intrinsic changes over time.

Note that this implies that the correspondence between internal and external states must be learned, i.e. our preferences are not simply given to us but acquired with experience, and that we might be uncertain about whether we are hungry, tired or happy. This relates to the framework of "active inference", under which reward values are inferred rather than being directly conferred [17]. We conceive of this internal state space as a multidimensional map whose dimensions variously pertain to both physiological states (e.g. satiety) and cognitive goals (e.g. social status). Internal states are subject to momentum via recurrent connections so that states (or moods) may persist in the face of a change in external circumstance.

Second, the agent represents a set of desired (or aversive) points $\boldsymbol{\theta}^{*}$ on this space, allowing it to estimate the distance (and contributing features) of its current position to the goal state. In doing so, the agent needs to combine across the relevant goal dimensions and weight these according to their relative contribution to this distance by computing the Minkowski distance:

$$
\delta_{t+1}=\left\|\theta_{t+1}-\theta^{*}\right\|_{k}=\sqrt[p]{\sum\left|\theta_{t+1}-\theta^{*}\right|^{q}}, \text { for } p=q
$$

The parameter $k$ allows the agent to prefer bundles that only consist of one single asset $(k<1)$, be indifferent between all bundles $(k=1)$ or prefer mixed bundles $(k>1)$. The model is thus well placed to account for commonly observed indifference curves between two or more economic goods. If we further assume that there are two non-linearities, $p$ and $q$, rather than just $k$, our model can further account for diminishing marginal utility, Fig. 2c. This computational step is directly inspired by a proposal in ref [18].

Finally, where, then, is value (or: reward) in our framework? Value arises naturally from our framework when the agent compares its current distance to its desired state to its previous distance, which summarizes that the agent has progressed on at least one of its goals since the last time-point:

$$
R_{t+1}=\delta_{t}-\delta_{t+1}
$$

Value is thus a summary of whether the agent is approaching or retreating from its goals. This model encapsulates situations in which multi-attribute objects need to be integrated into a single reward signal, such that $R=X \times U$ if $A_{\theta}=0$ and $\mathrm{k}=1$ in Eq 2, where $\boldsymbol{\theta}^{*}$ can account for reference points such as running averages over past choices.

Goal equilibrium theory readily incorporates other accounts of reference-dependent evaluation by adjusting how the agent computes $\theta^{*}$. For instance, it may do so by using a running average over past options and anchor its behaviour to this reference. When $k>1$, simply assuming that other drives exist that cannot currently be satisfied immediately gives rise to the ubiquitous phenomenon of diminishing marginal utility. In other words, as the agent acquires the currently relevant asset (such as liquid 
reward) its attention will progressively tend towards other relevant assets, diminishing the contribution of liquid reward to its well-being (Fig. 2c).

Our view relates to an earlier proposal that animals are motivated to satisfy a "current concern" and understands disorders of mental health as disrupted goal selection, pursuit and appraisal [36]. A fleshed out computational theory of this process will require the specification of how agents learn the relationship between individual states and the degree of goal realisation. One model proposes a distinction between "primary" and "learned" value, where the former resembles the traditional reward signal in the RL framework and the latter quantifies the eligibility of states for goal realisation [33]. A very appealing aspect of this framework is that it provides a natural way to understand the affective states that pervade our everyday mental landscape, including satisfaction (goal completion), frustration (goal obstruction), and disappointment (goal abandonment), which have largely eluded computational description thus far [33].

\section{A map of internal state in the OFC}

For valuation to depend on the internal state of the agent, these states must be explicitly represented in brain signals. However, most neural studies of value-guided choice have used a paradigm in which the animal or human participant makes a succession of unrelated, independent choices between food items or monetary gambles. This paradigm is not well suited to measuring how variation in internal state (how sated am I right now? How much wealth have I accumulated?) might be coded in neural circuits. However, recently researchers have begun to employ more complex paradigms that may shed some light on this issue.

At the neural level, the integrity of the orbitofrontal cortex (OFC) is critical for goal-driven reward behaviour [37]. Interestingly, a recent theory proposes that the OFC constitutes a "map" of both observable and latent states within a task [13,38], similar to the framework proposed here (c.f. Eq.1 in Box 2). Moreover, OFC may code endogenous (internal) as well as exogenous (external) value, as firing rates [39] and BOLD signals [40] can be choice-predictive even in the baseline period prior to stimulus onset. An emerging theory suggests that OFC encodes affective states that pertain to ongoing positive or negative value, i.e. moods or "momentum" in internal value states [41, 42]. Together, these findings imply that OFC is a likely candidate for representing ongoing estimates of position on the value map.

One recent study directly tested whether BOLD signals in OFC tracked the internal accumulation of financial resources across an episode in a way consistent with the ongoing internal state representation proposed here [43]. Participants performed an economic task that involved gambling for financial incentives over successive, independent trials. For a rational agent performing this task, there is no imperative to track the accumulated level of payoff incurred by choices - to maximise return, one simply needs to select, on each trial, the gamble with the highest expected value. However, when humans performed this gambling task over multiple discrete episodes, each signalled by a prominent contextual cue that was structurally irrelevant to the task, brain activity in the medial OFC tracked the level of accumulated resources (or "wealth") over the context (Fig. 3). This "value accumulation" signal occurred even though the accumulated rewards were never overtly signalled to participants, nor were they instructed or incentivised to calculate their ongoing wealth.

Another brain imaging study revealed that a nearby region of medial prefrontal cortex tracks internal states during wealth accumulation [44]. Participants were asked to choose among four alternatives (bandits) that either paid or charged an uncertain sum of money. Critically, each selected bandit was fungible - it continued to incur financial costs or benefits across a short block of trials, as if the agent had purchased that asset and was continually profiting (or otherwise) from its possession ("rule in" condition). In a different condition, the bandits began in the agent's possession and decisions had to 
be made about which (if any) bandits to "rule out". This allowed us to assess how neural encoding of value depended on whether an asset was being bought or sold. Critically, BOLD signals in the ventromedial prefrontal cortex (VmPFC) encoded the cumulative expected value of assets across the block (Fig. 3), and only coded for momentary rewards when decisions were made with future consequences for reward (e.g. when a bandit was selected in the "rule in" condition, or not selected in the "rule out" condition). Together, these studies suggest that the reward system tracks level of accumulated resources over time, for example so that future decision policies can adapt according to the current internal state, in a way that is compatible with goal equilibrium theory.

\section{Budget rules: aspiration and avoidance points}

The natural world is structured in such a way that some states are critical for survival or have substantial impact on long-run future outcomes. For example, a student might work hard to pass his exams in the knowledge that it will open up interesting career opportunities. These states are often attained when accumulated resources reach, or fall below, a critical threshold. Behavioural ecologists have argued that animals' risky foraging behaviour adapts to satisfy a "budget rule" that seeks to maintain energetic resources at aspirational levels that safely offset future scarcity. For example, birds make risky foraging choices at dusk in order to accrue sufficient energy to survive a cold night [45]. This view is neatly accommodated within the framework proposed here, in that the aspiration level reflects the setpoint against which current resource levels are compared, and the driver of behaviour is the disparity between current state and goal.

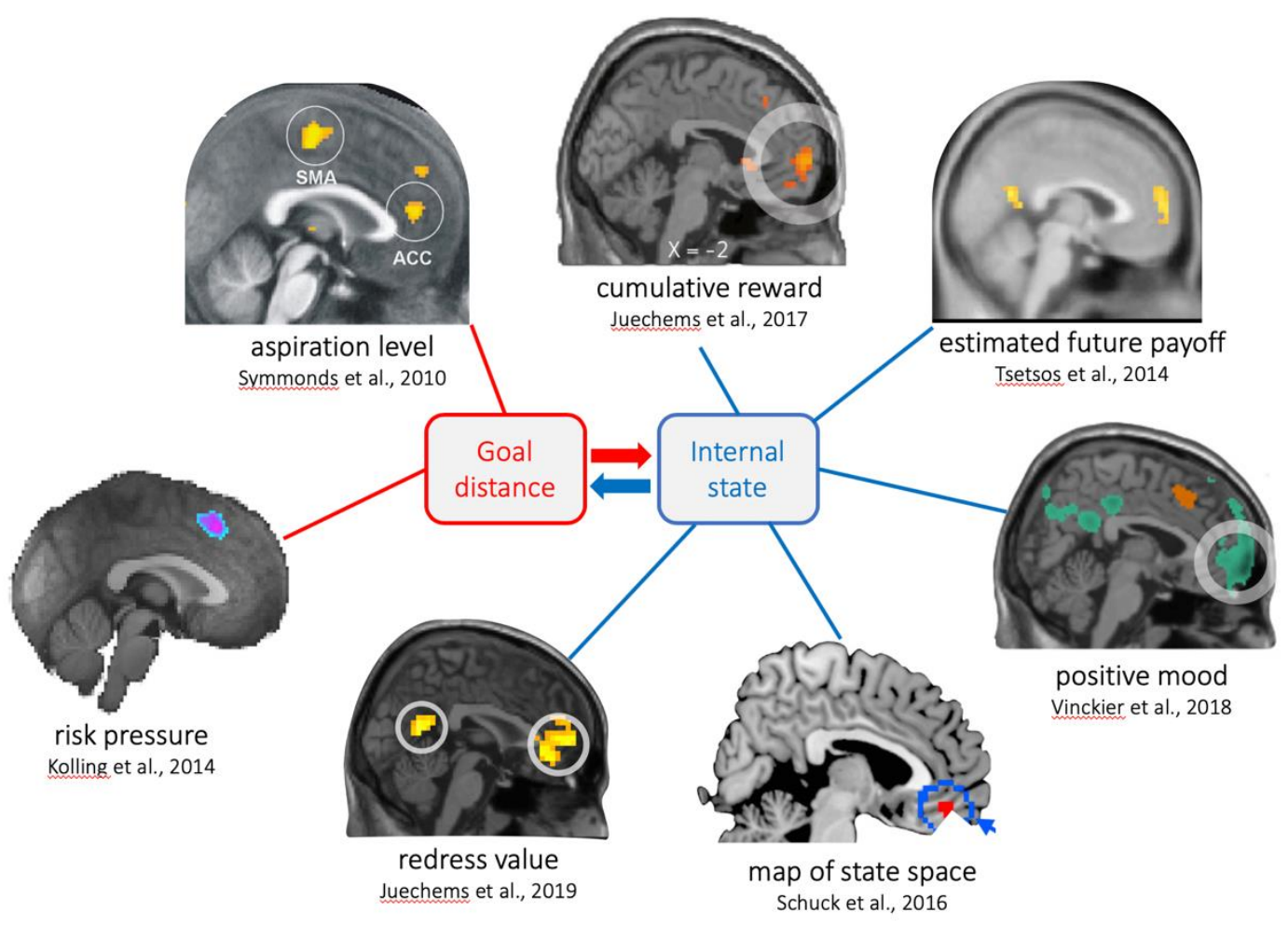

Figure 3. Cortical areas on the medial surface correlating with diverse aspects of internal state and goal distance. Reprinted with permission [pending] from refs $[2,43,44,46,47]$.

Recent work with brain imaging $[46,47]$ suggests that this goal distance signal might be computed in a different medial prefrontal cortex region, the dorsal anterior cingulate cortex (dACC). Symmonds [46] asked participants to make a series of gambles which only paid out if a critical threshold or aspiration 
point was reached. BOLD signals in the VmPFC scaled with the expected future return over a series of gambles, similar to [44], whereas dACC coded for the aspiration point itself (Fig. 3). Using a very similar design, Kolling [47] asked human participants to accept or reject monetary gambles over a short block, with cumulative earnings increased by a multiplier if they reached a fixed aspiration level. BOLD signals in the dACC coded for number of steps to goal, as well as the discrepancy between accumulated return and the aspiration level, scaled by the distance to the end of the block. This signal, which the authors call "risk pressure", seems to be signalling the agent's current position with respect to a goal, rather than merely the arrival of a punctate reward. In another study involving planning in a virtual subway environment, dACC BOLD signals coded for the distance to goal in number of context switches [48]. Indeed, the dACC is one brain region where single-cell responses gradually build up in association with a series of actions that precede reward delivery [49], and where lesions provoke failures of task persistence [50], as if this region participated more generally computing the disparity signal $\theta^{*}-\theta$ in the service of goal-directed behaviour.

A
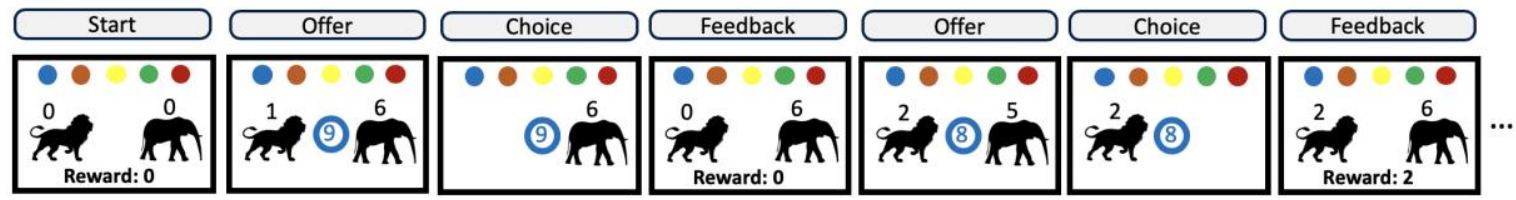

B

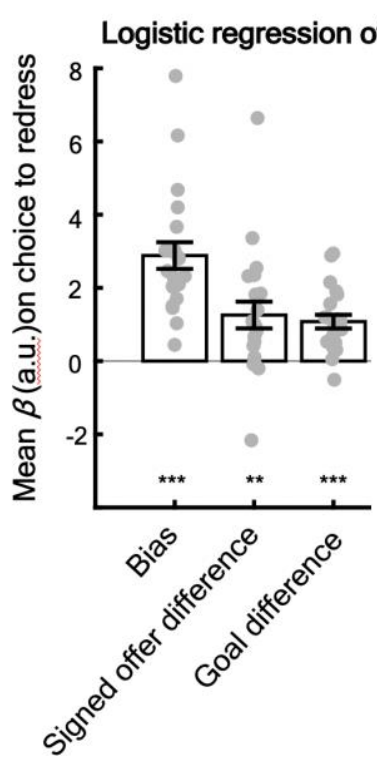

C
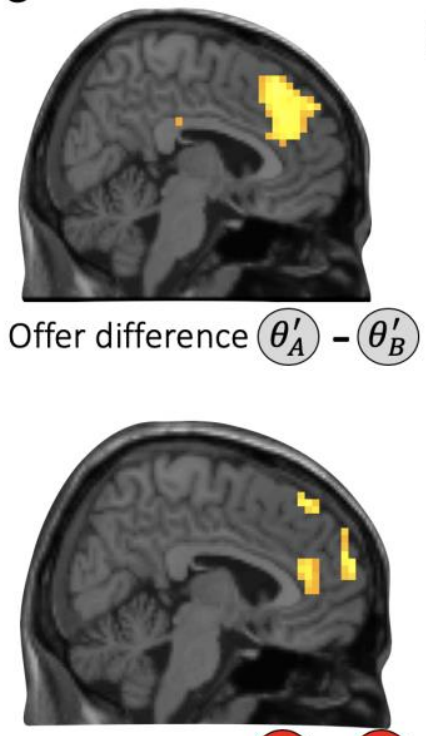

Goal difference $\theta_{Y}-\theta_{X}$
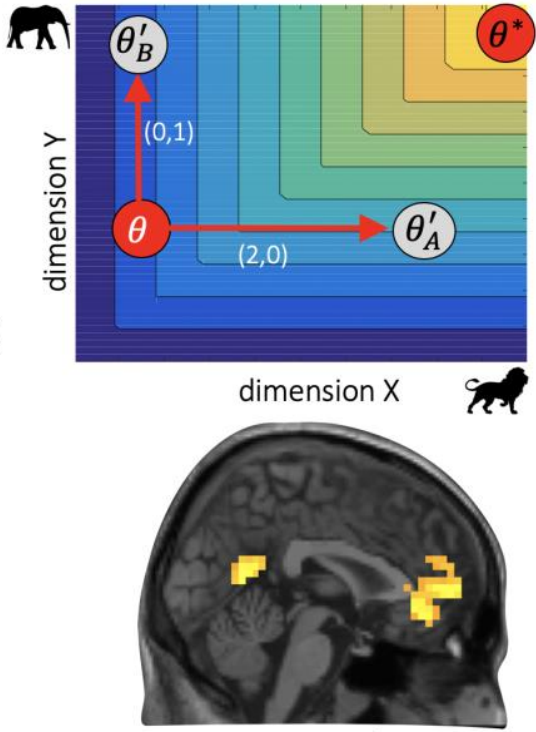

Redress value $\frac{d}{d T} \theta$

Figure 4. Neural signals for computing goal equilibrium. A. Experimental task used in Juechems et al., 2019. Participants managed virtual zoos in which they needed to accumulate as many lions and elephants as possible. Starting out from an empty zoo, participants were offered varying amounts of lions and elephants to acquire. Importantly, reward was calculated as the lower tally of animals currently owned within the zoo (e.g. when participants owned 2 lions and 6 elephants, they earned 2 units of reward). This enforced a rule by which the two tallies should be held approximately in equilibrium across time. Participants completed five zoos per block with their respective length indicated as a countdown in the centre of the screen. B. Logistic regression predicting a "redress" choice - i.e. whether participants chose to increase the lower tally of animals currently in the zoo (as given by the feedback screen). Participants placed approximately equal weight on the offer and goal (tally) differences, as in the simulation in Fig. 2c. C. Indifference map over the two assets (lions and elephants) and three key neural correlates. Regions in the dACC and vmPFC encoded the offer difference, goal difference, and the redress (the decrease in distance to goal between trials). Reprinted with permission [pending] from ref [2]. 
In the standard RL framework, each state-action pair is associated with a scalar value, often called a Qvalue [1]. In other words, most RL models assume that value is a unidimensional quantity (although "multi-objective" RL models relax this assumption [51]). This seems incongruous when considering the challenges that animals face in natural environments, which involve jointly satisfying many different constraints (for example, maintaining levels of satiety, hydration, warmth and social capital). Rewards take many different forms - from food items to social and sexual signals to secondary reinforcers such as money. A popular view in the hybrid field of neuroeconomics is that neural signals allow potentially incommensurable rewards to be encoded in a single neural "common currency" [31]. For example, single cells in OFC code for the "offer value" of different liquid rewards irrespective of their taste [52]. The existence of a common neural code for different assets allows economists to understand rational decision-making in consumer settings, which might involve choosing between a meal in a restaurant or tickets to the theatre. However, goal equilibrium theory suggests a different perspective, whereby assets are explicitly encoded along different dimensions (needs) in the value map, and decisions are made according to whichever need is currently greatest.

Without further assumptions, goal equilibrium theory predicts that animals will treat different assets as if they are imperfect substitutions for one another. Multidimensional prospects (or "bundles") should be preferred if they contain a mixture of different assets, because this allows multiple drives to be satisfied simultaneously (Fig. 2b). For example, it is better to be neither too hungry nor too thirsty than to be starving or dehydrated. Several longstanding empirical results support this view. First of all, in early conditioning experiments it was observed that a cue that is associated either simultaneously or successively with multiple assets - such as food and water - has greater value that an equivalent cue associated with only a single asset (or drive) [53]. Finally, a preference for compromise decisions is also apparent from studies of so-called "decoy" effects in multi-attribute, multi-alternative choices. Faced with a choice between two iso-preferred items that have complementary costs and benefits (e.g. an expensive but high quality consumer product $A$, or a cheaper but lower quality product $B$, the introduction of a "decoy" that is higher in price/quality than A will bias preferences towards A, whereas an item that is lower in price/quality than B will bias choices towards B, as if participants had an intrinsic preference for the compromise solution [54]. This preference is predicted by the goal equilibrium model by assuming that agents aspire to high quality and low price simultaneously, rendering the agent's indifference curve over options convex (Fig. 2b).

In natural environments we are faced with choices between offers of different quantity and quality, in variable states of need. In such scenarios, our model predicts that both goal difference (the relative level of two internal resources $A$ and $B$ ) and offer difference (the relative quality of offer of $A$ and $B$ ) will predict choices, Fig. 2c. This prediction was tested in a recent study in which participants made decisions that directly trade off multiple assets. Juechems [2] asked participants to perform a task that involved managing a virtual zoo containing lions and elephants. On each trial they populated the zoo by choosing among offers of variable numbers of each animal, and the financial success of their zoo was proportional to the minimum number of either lions or elephants present on each trial (which was not directly observed), Fig. 4a. Each choice was classified according to whether it "redressed" the imbalance in cumulative lions and elephants and decisions to "redress" were predicted by both goal difference and offer difference, Fig. 4b. Critically, BOLD signals in the VMPFC signalled the level of redress incurred by a choice - the extent to which a choice brought the numbers of animals into equilibrium, but independent of the total animal numbers and thus of any overall financial return. Interestingly, the $\mathrm{dACC}$ coded for the level of imbalance among assets, i.e. the pressure to redress this imbalance, reminiscent of the "risk pressure" signal described above [47], Fig. 4c. 
533

534

\section{Concluding Remarks}

Our contribution here is twofold. Firstly, we highlight some challenges for RL as a computational theory of motivated behaviour in biological agents. The RL framework assumes that rewards originate in the environment, that they are sensed by dedicated input channels, that they are not fungible, and (typically) unidimensional. Secondly, we propose an alternative framework, "goal equilibrium theory" that treats reward as the by-product of computing distance to largely self-defined (intrinsic) goals. In doing so, we acknowledge a debt to theories of motivation past $[20,36]$ and present $[18,33]$.

Although our theory is preliminary, its readily accounts for commonly observed effects in human and animal behaviour, such as reward-maximization, reference-dependence, diminishing marginal utility, and convex indifference between goods. Empirical tests of the theory's wider predictions will require tasks (or field observations) that involve accumulation of multiple assets. Such tasks are rare in the literature concerning value-guided choices, and where multiple value dimensions inadvertently come into play, they are often even discarded as nuisance variables. Goal equilibrium theory may provide a framework in which to probe goal-directed behaviour using richer tasks which more closely emulate the fact that human (as well as animal) behaviour is driven by multiple, competing goals.

\section{Box 3. Outstanding questions.}

How do we form and learn about new goals during development and maturation, and how are these incorporated into the value map?

How does the notion of a "value map" proposed here relate to theories proposing cognitive maps in other domains, such as spatial navigation and conceptual spaces?

Distance to goal depends on policy. How is the agent's policy factored into the goal distance computation?

What is the precise division of labour between the VMPFC and $\mathrm{AACC}$ in representing internal states and computing goal distance?

Goal realisation may be uncertain. How is the probability of goal attainment, i.e. the "feasibility function" represented in neural circuits?

Can we capture a wide range of psychiatric disorders, such as addiction or mood disorders, using goal equilibrium theory by adjusting how much distinct goals compete? For example, could a lowered tendency to discard unrealistic goals account for sustained negative mood?

\section{Highlights}

- The computational framework of Reinforcement Learning has allowed us to make great strides in understanding a wide range of motivated behaviours, as well as permitting us to build intelligent artificial agents

- However, RL does not explain the dependence of value-guided choices on ongoing needs, affective states, or abstract goals.

- Recent neural evidence suggests that areas in the medial prefrontal cortex represent internal value states and distance to intrinsic goals in a way that is not readily explained by the RL framework

- We propose that reward emerges from the more general mechanism of computing distance to intrinsic goals and that this framework can explain a range of behavioural and neural data from value-guided decision tasks in humans and other animals. 
546 1. Sutton, R. and Barto, A. (1998) Reinforcement Learning, MIT press.

547 2. Juechems, K. et al. (2019) A Network for Computing Value Equilibrium in the Human Medial

548 Prefrontal Cortex. Neuron 101 (5), 977-987 e3.

549 3. Dayan, P. and Daw, N.D. (2008) Decision theory, reinforcement learning, and the brain. Cogn

550 Affect Behav Neurosci 8 (4), 429-53.

551 4. Dolan, R.J. and Dayan, P. (2013) Goals and habits in the brain. Neuron 80 (2), 312-25.

552 5. Schultz, W. et al. (1997) A neural substrate of prediction and reward. Science 275 (5306), 1593-9.

553 6. Mnih, V. et al. (2015) Human-level control through deep reinforcement learning. Nature 518

554 (7540), 529-33.

555 7. Silver, D. et al. (2017) Mastering the game of Go without human knowledge. Nature 550 (7676),

556 354-359.

557 8. Maslow, A.H. (1943) A theory of human motivation. Psychological Review 50, 370-96.

558 9. Fadel, J. and Deutch, A.Y. (2002) Anatomical substrates of orexin-dopamine interactions: lateral hypothalamic projections to the ventral tegmental area. Neuroscience 111 (2), 379-87. 10. Schultz, W. (2015) Neuronal Reward and Decision Signals: From Theories to Data. Physiol Rev 95 (3), 853-951.

11. Oudeyer, P. and Kaplan, F. (2007) What is Intrinsic Motivation? A Typology of Computational Approaches. Front Neurorobotics 1:6.

12. Ryan, R.M. and Deci, E.L. (2000) Intrinsic and Extrinsic Motivations: Classic Definitions andNew Directions. Contemporary Educational Psychology 25, 54-67.

13. Schuck, N.W. et al. (2016) Human Orbitofrontal Cortex Represents a Cognitive Map of State Space. Neuron 91 (6), 1402-1412.

14. Gottlieb, J. and Oudeyer, P.Y. (2018) Towards a neuroscience of active sampling and curiosity. Nat Rev Neurosci 19 (12), 758-770.

15. Guo, X. et al. (2016) Deep Learning for Reward Designto Improve Monte Carlo Tree Search in ATARI Games. arXiv.

16. Friston, K. (2010) The free-energy principle: a unified brain theory? Nat Rev Neurosci 11 (2), 12738.

17. Pezzulo, G. et al. (2015) Active Inference, homeostatic regulation and adaptive behavioural control. Prog Neurobiol 134, 17-35.

18. Keramati, M. and Gutkin, B. (2014) Homeostatic reinforcement learning for integrating reward collection and physiological stability. Elife 3.

578 19. Berridge, K.C. (2004) Motivation concepts in behavioral neuroscience. Physiol Behav 81 (2), 179579209.

580 20. Hull, C.L. (1943) Principles of Behavior: An Introduction to Behavior Theory, Appleton-Century581 Croft.

582 21. N.E., M. and M.L., K. (1952) Reward effects of food via stomach fistula compared with those of 583 food via mouth. J Comp Physiol Psychol 45, 555-564.

584 22. Olds, J. and Milner, P. (1954) Positive reinforcement produced by electrical stimulation of septal 585 area and other regions of rat brain. Journal of Comparative and Physiological Psychology 47, 419-

586427.

587 23. McNamara, J.M. et al. (2012) The ecological rationality of state-dependent valuation. Psychol 588 Rev $119(1), 114-9$.

589 24. Mollenauer, S.O. (1971) Shifts in deprivation level: different effects depending on the amount of 590 preshift training. Learning and Motivation 2, 58-66.

25. Pompilio, L. and Kacelnik, A. (2005) State-dependent learning and suboptimal choice: When starlings prefer long over short delays to food. Animal Behaviour 70, 571-578.

26. Arieli, A. et al. (1996) Dynamics of ongoing activity: explanation of the large variability in evoked cortical responses. Science 273 (5283), 1868-71. 
27. Allen, W.E. et al. (2017) Thirst-associated preoptic neurons encode an aversive motivational drive. Science 357 (6356), 1149-1155.

28. Allen, W.E. et al. (2019) Thirst regulates motivated behavior through modulation of brainwide neural population dynamics. Science.

29. Rushworth, M.F. et al. (2011) Frontal cortex and reward-guided learning and decision-making. Neuron 70 (6), 1054-69.

30. Rangel, A. et al. (2008) A framework for studying the neurobiology of value-based decision making. Nat Rev Neurosci 9 (7), 545-56.

31. Kable, J.W. and Glimcher, P.W. (2009) The neurobiology of decision: consensus and controversy. Neuron 63 (6), 733-45.

32. Glimcher, P.W. (2004) Decision, Uncertainty and the Brain: : The Science of Neuroeconomics, MIT Press.

33. O'Reilly, R.C. et al. (2014) Goal-Driven Cognition in the Brain: A Computational Framework. arXiv arXiv:1404.7591.

34. Knox, R.E. and Inkster, J.A. (1968) Postdecision dissonance at post time. Journal of Personality and Social Psychology 8, 319-323.

35. Daw, N.D. et al. (2005) Uncertainty-based competition between prefrontal and dorsolateral striatal systems for behavioral control. Nat Neurosci 8 (12), 1704-11.

36. Klinger, E. (1975) Consequences of Commitment to and Disengagement from Incentives. Psychol Rev 82, 1-25.

37. Burke, K.A. et al. (2008) The role of the orbitofrontal cortex in the pursuit of happiness and more specific rewards. Nature 454 (7202), 340-4.

38. Wilson, R.C. et al. (2014) Orbitofrontal cortex as a cognitive map of task space. Neuron 81 (2), 267-79.

39. Padoa-Schioppa, C. (2013) Neuronal origins of choice variability in economic decisions. Neuron 80 (5), 1322-36.

40. Abitbol, R. et al. (2015) Neural mechanisms underlying contextual dependency of subjective values: converging evidence from monkeys and humans. J Neurosci 35 (5), 2308-20.

41. Eldar, E. et al. (2016) Mood as Representation of Momentum. Trends Cogn Sci 20 (1), 15-24.

42. Vinckier, F. et al. (2018) Neuro-computational account of how mood fluctuations arise and affect decision making. Nat Commun 9 (1), 1708.

43. Juechems, K. et al. (2017) Ventromedial Prefrontal Cortex Encodes a Latent Estimate of Cumulative Reward. Neuron 93 (3), 705-714 e4.

44. Tsetsos, K. et al. (2014) Neural mechanisms of economic commitment in the human medial prefrontal cortex. Elife 3.

45. Caraco, T. (1981) Energy budgets, risk and foraging preferences in dark-eyed juncos (Junco hyemalis). Behav Ecol Sociobiol. 8, 213-217.

46. Symmonds, M. et al. (2010) A behavioral and neural evaluation of prospective decision-making under risk. J Neurosci 30 (43), 14380-9.

47. Kolling, N. et al. (2014) Multiple neural mechanisms of decision making and their competition under changing risk pressure. Neuron 81 (5), 1190-1202.

48. Balaguer, J. et al. (2016) Neural Mechanisms of Hierarchical Planning in a Virtual Subway Network. Neuron 90 (4), 893-903.

49. Shidara, M. and Richmond, B.J. (2002) Anterior cingulate: single neuronal signals related to degree of reward expectancy. Science 296 (5573), 1709-11.

50. Kennerley, S.W. et al. (2006) Optimal decision making and the anterior cingulate cortex. Nat Neurosci 9 (7), 940-7.

51. Liu, C. et al. (2014) Multiobjective Reinforcement Learning: A Comprehensive Overview. IEEE Transactions on Systems, Man, and Cybernetics: Systems 45, 385 - 398.

52. Padoa-Schioppa, C. and Assad, J.A. (2006) Neurons in the orbitofrontal cortex encode economic value. Nature 441 (7090), 223-6. 
53. Wike, E.L. and Barrientos, G. (1958) Secondary reinforcement and multiple drive reduction.

647 Journal of Comparative and Physiological Psychology 51, 640-643.

648 54. Simonson, I. (1989) Choice Based on Reasons: The Case of Attraction and Compromise Effects. 649 Journal of Consumer Research 16, 158-174.

650

651 